\title{
A Correction Scheme for Fano Line Shapes in Two-Dimensional Infrared Spectroscopy
}

\author{
Tek, Gökçen ; Hamm, Peter
}

\begin{abstract}
The asymmetry of Fano line shapes observed for metal-adsorbate systems is reflected in twodimensional infrared (2D IR) spectroscopy as a distorted spectrum. A phenomenological correction scheme is proposed that transforms distorted 2D IR spectra into conventional spectra. To that end, a phase correction factor is first derived from the IR absorption spectrum of the sample by symmetrizing the asymmetric line shape and subsequently applied to the distorted 2D IR spectra. The concept is illustrated for a model system consisting of an organic molecule (p-mercaptobenzonitrile) adsorbed on a sputter-coated metal layer $(\mathrm{Au})$. The correction scheme reveals conventional, easily interpretable 2D IR spectra.
\end{abstract}

DOI: https://doi.org/10.1021/acs.jpclett.0c01752

Posted at the Zurich Open Repository and Archive, University of Zurich ZORA URL: https://doi.org/10.5167/uzh-198692

Journal Article

Accepted Version

Originally published at:

Tek, Gökçen; Hamm, Peter (2020). A Correction Scheme for Fano Line Shapes in Two-Dimensional Infrared Spectroscopy. Journal of Physical Chemistry Letters, 11(15):6185-6190.

DOI: https://doi.org/10.1021/acs.jpclett.0c01752 


\title{
A Correction Scheme for Fano Line Shapes in Two-Dimensional Infrared Spectroscopy
}

\author{
Gökçen Tek, Peter Hamm \\ Department of Chemistry, University of Zurich, Zurich, Switzerland \\ corresponding author: peter.hamm@chem.uzh.ch \\ (Dated: July 17, 2020)
}

\begin{abstract}
The asymmetry of Fano lineshapes observed for metal-adsorbate systems is reflected in two-dimensional infrared (2D IR) spectroscopy as a distorted spectrum. A phenomenological correction scheme is proposed that transforms distorted 2D IR spectra into a conventional one. To that end, a phase correction factor is first derived from the IR absorption spectrum of the sample by symmetrizing the asymmetric lineshape, and subsequently applied to the distorted 2D IR spectra. The concept is illustrated for a model system consisting of an organic molecule (paramercaptobenzonitrile) adsorbed on a sputter-coated metal layer ( $\mathrm{Au}$ ). The correction scheme reveals conventional, easily interpretable 2D IR spectra.
\end{abstract}

TOC Graphic

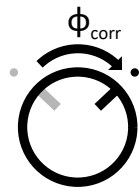


Optical properties of molecules are modified when adsorbed on metal nanostructures, since the interaction of electromagnetic (EM) radiation causes coherent oscillations of free electrons in those nanostructures (localized surface plasmons). When the excitation wavelength is larger than the size of the particles, to which the plasmons are confined, the field is enhanced in the vicinity of the particle. ${ }^{1}$ Vibrational dipoles of molecules experiencing this near-field enhancement lead to an effect called surface-enhanced infrared absorption (SEIRA). Since the near-field enhancement is concentrated within a few nanometers from the metallic nanostructures, ${ }^{2}$ SEIRA enables highly sensitive infrared detection of vibrational modes at interfaces. One distinguishes two types of SEIRA spectroscopy, nonresonant and resonant SEIRA. ${ }^{3}$ Nonresonant SEIRA refers to randomly distributed metal particles or islands, ${ }^{4}$ which show a broad resonance that extends to mid-IR frequencies. ${ }^{5,6}$ On the other hand, resonant SEIRA involves tailored nanostructures (e.g., nanoantennas),${ }^{7-9}$ where the plasmonic resonance can be tuned in the desired frequency range for the signal enhancement. ${ }^{10}$ In comparison to metal islands, significantly higher signal enhancement and spectral selectivity is achieved by nanoantennas.

Recently our group, as well as others, have combined SEIRA spectroscopy with two-dimensional infrared (2D IR) spectroscopy. ${ }^{8,11-14}$ By using sputtered metal layers, we have shown significant enhancement in the non-linear signal from a monolayer of a weak IR absorber ${ }^{11}$ and have achieved vibrational ladder climbing. ${ }^{13}$ On the other hand, we have shown that asymmetric lineshapes (lineshape distortions) arise when a certain metal thickness is exceeded. Similar asymmetric lineshapes were also observed in the 2D IR spectra of nanoantennas. ${ }^{8,9,14}$

The asymmetric lineshape is the result of an interference between two fields, which are phase shifted with respect to each other, and which originate from a discrete state and a coupled continuum of states. The phenomenon has been described by Fano, ${ }^{15}$ who formulated for the first time what later became known as "Fano lineshape":

$$
f_{\text {Fano }}(\Omega)=\frac{\left(q_{\text {Fano }}+\Omega\right)^{2}}{1+\Omega^{2}} .
$$

Here, $q_{\text {Fano }}$ is the Fano asymmetry parameter and $\Omega=$ $2\left(\omega-\omega_{0}\right) / \gamma$ the reduced frequency with $\omega_{0}$ the resonance frequency of the transition and $\gamma$ its linewidth. Fano lineshapes are found in very different physical situations. For example, in Fano's original paper, ${ }^{15}$ it is the interference of a discrete electronic state of He with the ionisation continuum. In SEIRA spectroscopy, Fano lineshapes are the result of vibrational transitions interfering with the plasmon resonance. ${ }^{16-19}$ The theory has been worked out for both nonresonant ${ }^{3,17,19-22}$ and resonant ${ }^{10,23,24}$ SEIRA spectroscopy. Fano lineshapes were also observed on ordered crystalline surfaces due to the coupling of electronhole pairs and adsorbate vibrations. ${ }^{25-29}$ A somewhat different example, also leading to distorted lineshapes, con- cerns the resonance Mie mechanism in diffuse reflection spectroscopy. ${ }^{30}$

While Eq. 1 is most commonly used in the context of the type of phenomena listed above, it is easy to show that Eq. 1 is mathematically identical to a phase-shifted Lorentzian lineshape function (apart from an offset and a scaling factor):

$$
f_{\text {Lorentz }}(\Omega)=\Re\left(\frac{e^{i \phi}}{1-i \Omega}\right),
$$

with $\cot (\phi / 2)=-q_{\text {Fano. }}$. Ref. 31 has exploited that relationship to optically correct Fano lineshapes, and we will do so computationally.

Compared to IR absorption spectroscopy, ultrafast 2D IR spectroscopy can provide significantly more information, such as (i) the coupling between vibrational modes by the appearance of cross-peaks, (ii) the structural distributions by the analysis of both homogeneous and inhomogeneous linewidths, (iii) the structural dynamics by time-dependent analysis of the center line slope, (iv) the potential energy surface by extracting the anharmonic shift, and (v) the surface enhancement factor for metals and the transition dipole moment of adsorbates by the analysis of the signal intensity from the 2D IR spectra. ${ }^{32}$ In this regard, asymmetric lineshapes are undesirable features that make analysis of 2D IR spectra complicated. Until now, we have avoided this issue by either using ultra-thin layers with no lineshape asymmetry, ${ }^{33,34}$ or by tuning the metal thickness and the angle of incidence of the beam to invert the sign of the lineshape. ${ }^{13}$ Such restrictions pose a limit in achieving high enhancement factors and undistorted spectra concurrently.

The theory of Fano-lineshapes has been extended to also explain distorted 2D IR spectra. ${ }^{14,35}$ Although such models help to gain insight into the physical mechanism, there exists no simple way to interpret distorted 2D IR spectra. This is in particular true when dealing with a rough (sputtered) metal layer, whose geometry is not well defined, unlike that of nanoantennas. Such metal layers are very common in photo/electrocatalysis. ${ }^{36}$

Here, we present a phenomenological correction scheme that enables the transformation of a distorted 2D IR spectrum into a conventional one, which then can be interpreted in the usual way. ${ }^{32}$ Our scheme involves two steps: the retrieval of a phase factor from an IR absorption spectrum and the subsequent application of the obtained phase factor to 2D IR spectra. For an illustration of the correction scheme, we have chosen paramercaptobenzonitrile $(p-\mathrm{PhCN})$ immobilized on sputtercoated $\mathrm{Au}$ layers $(\mathbf{A u}-\mathbf{C N})$ with varying metal thickness as model system (Figure 1c). To avoid further complications on the lineshape in the ATR geometry, ${ }^{37}$ utilized in our previous work on the same molecular system, ${ }^{13}$ we used a transmission geometry here.

Fig. 1a shows IR absorption spectra of Au-CN for metal thicknesses of 2.5, 3.5, 4 and $4.5 \mathrm{~nm}$. The adsorbate bands are superposed on a broad background absorption, which increases with the metal layer thickness 


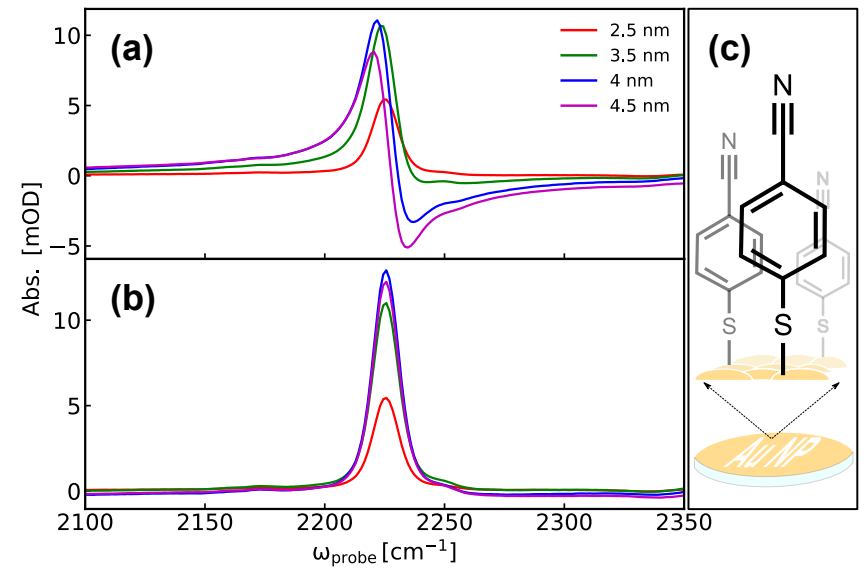

FIG. 1. IR absorption spectra of 2.5, 3.5, 4 and $4.5 \mathrm{~nm} \mathrm{Au-}$ CN (a) before and (b) after applying the correction scheme. Metal offsets of each spectra in (a) were subtracted from the raw data (see Figure S1 in the Supporting Information). (c) Illustration of the Au-CN system.

(see Figure S1a,b in the Supporting Information), and which has been subtracted in Fig. 1a. The background absorption originates from the randomly distributed plasmon resonances from metal islands with different sizes and geometries. ${ }^{3,7,10}$ With increasing thickness, metal particles start to aggregate, causing the resonance to red-shift and broaden due to the interaction of induced dipoles or higher multipoles. ${ }^{38} \mathbf{A u}-\mathbf{C N}$ on a $2.5 \mathrm{~nm}$ layer shows a symmetric lineshape with central frequency and linewidth of $\sim 2225 \mathrm{~cm}^{-1}$ (CN stretch) and $\sim 13 \mathrm{~cm}^{-1}$, respectively. As the metal layer becomes thicker, the lineshape becomes increasingly more asymmetric (Fig. 1a).

To retrieve the phase factor that transforms the asymmetric lineshape into a symmetric one, we make use of the Kramers-Kronig relation. That is, the measured spectrum $A(\omega)$ is real-valued, but the complementary imaginary part can be retrieved by enforcing causality in the time-domain in a Hilbert transformation. ${ }^{39}$ To that end, we first Fourier-transformed $A(\omega)$ into the time-domain, zeroed negative times to enforce causality, and Fouriertransformed back into the frequency domain:

$$
\begin{aligned}
A(\omega) & \stackrel{\mathcal{F} \mathcal{T}^{-1}}{\longrightarrow} \tilde{A}(t) \rightarrow \Theta(t) \tilde{A}(t) \\
& \stackrel{\mathcal{F} \mathcal{T}}{\longrightarrow} \tilde{A}(\omega) \stackrel{2 \Re\left(\tilde{A}(\omega) e^{i \phi}\right)}{\longrightarrow} A_{\phi}(\omega)
\end{aligned}
$$

where $\Theta(t)$ is the Heaviside step function. In the final step, we calculated a linear combination of real and imaginary parts of $\tilde{A}(\omega)$ by adding a phase factor (multiplication by 2 was needed because half of the data were discarded after the inverse Fourier-transformation). The phase $\phi$ was then varied to find the most symmetric lineshape. To that end, we first found the frequency, $\omega_{0}$, of the band maximum with the help of a spline interpolation, and defined a symmetric grid around that point in a range that roughly equals twice the band-width of the line. We then minimized:

$$
\sum_{k=1}^{n}\left|A_{\phi}\left(\omega_{k}\right)-A_{\phi}\left(\omega_{-k}\right)\right|
$$

with respect to $\phi$, where $-\pi<\phi<\pi$ was restricted to a range in which $A_{\phi}\left(\omega_{0}\right)>0$.

Figure $1 \mathrm{~b}$ shows the corrected IR spectra with values $\phi_{\text {corr }}=0.4,0.9$ and $1.23 \mathrm{rad}$ for $3.5,4$ and $4.5 \mathrm{~nm}$ Au-CN, respectively. Voigt lineshape fits to all corrected spectra resulted in $\sim 2225 \mathrm{~cm}^{-1}$ and $\sim 13 \mathrm{~cm}^{-1}$ for the central frequencies and linewidths, respectively. These values are the same as those obtained for $2.5 \mathrm{~nm}$ $\mathbf{A u}-\mathbf{C N}$, where no correction is needed. Furthermore, in contrast to the uncorrected spectra (Figure 1a), the band intensities can be compared directly in the corrected spectra. The absorbance increases until $4 \mathrm{~nm}$ $\mathbf{A u}-\mathbf{C N}$, as a consequence of signal enhancement, and subsequently decreases again at $4.5 \mathrm{~nm} \mathbf{A u}-\mathbf{C N}$. When the metal particles start to aggregate, the enhancement is the strongest in the voids between adjacent metal islands due to crowding of the EM field lines. ${ }^{33,40}$ Beyond a certain thickness that effect stops because of a loss of the island-like feature. ${ }^{41}$ Fig. S1c,d (Supporting Information) shows that the phase factor retrieved from the - CN mode at $2225 \mathrm{~cm}^{-1}$ band properly phases the complete spectral range from $\sim 1200 \mathrm{~cm}^{-1}$ to $\sim 1800 \mathrm{~cm}^{-1}$. This result emphasizes that the plasmon resonance is very broad and strongly smeared out for the sputtered metal surfaces considered here, with only a weak frequency-dependence of the phase.

We move on to the second step of the correction scheme, which involves the application of $\phi_{\text {corr }}$ to the $2 \mathrm{D}$ IR spectra. We measure 2D IR spectroscopy in pumpprobe geometry, ${ }^{42-44}$ see Materials and Methods for details. A 2D IR spectrum can be thought of as a series of pump-probe (PP) spectra stacked along a $\omega_{\text {pump }}$ axis, each excited by a different pump-spectrum, or, a series of absorption spectra of molecules that happen to be excited. The phase correction therefore was applied to the $\omega_{\text {probe }}$ axis by taking cuts along the $\omega_{\text {probe-axis for each }}$ $\omega_{\text {pump }}$, and treating each cut according to Eq. 3 with $\phi=\phi_{\text {corr }}$.

We start with discussing the 2D IR spectrum of $2.5 \mathrm{~nm}$ $\mathbf{A u}-\mathbf{C N}$, in which case the metal layer is thin enough so that no correction was needed for the IR absorption spectrum (Fig. 1a). The corresponding 2D IR spectrum at population time 0.3 ps shown in Figure 2c resembles what is expected for a 2D IR spectrum, with negative contributions from the ground state bleach (GSB)/stimulated emission (SE) on the diagonal and positive, excited state absorption (ESA) just below the GSB/SE signal.

To illustrate the correction scheme, we chose $4.5 \mathrm{~nm}$ Au-CN, since its IR spectrum showed the most asymmetric lineshape. Figure 2a shows the uncorrected 2D IR spectrum, after the removal of a constant offset arising from the excitation of the metal layer (see Figure S2 in the Supporting Information). The major difference, as compared to the 2D IR spectrum of $2.5 \mathrm{~nm} \mathrm{Au}-\mathbf{C N}$, is 


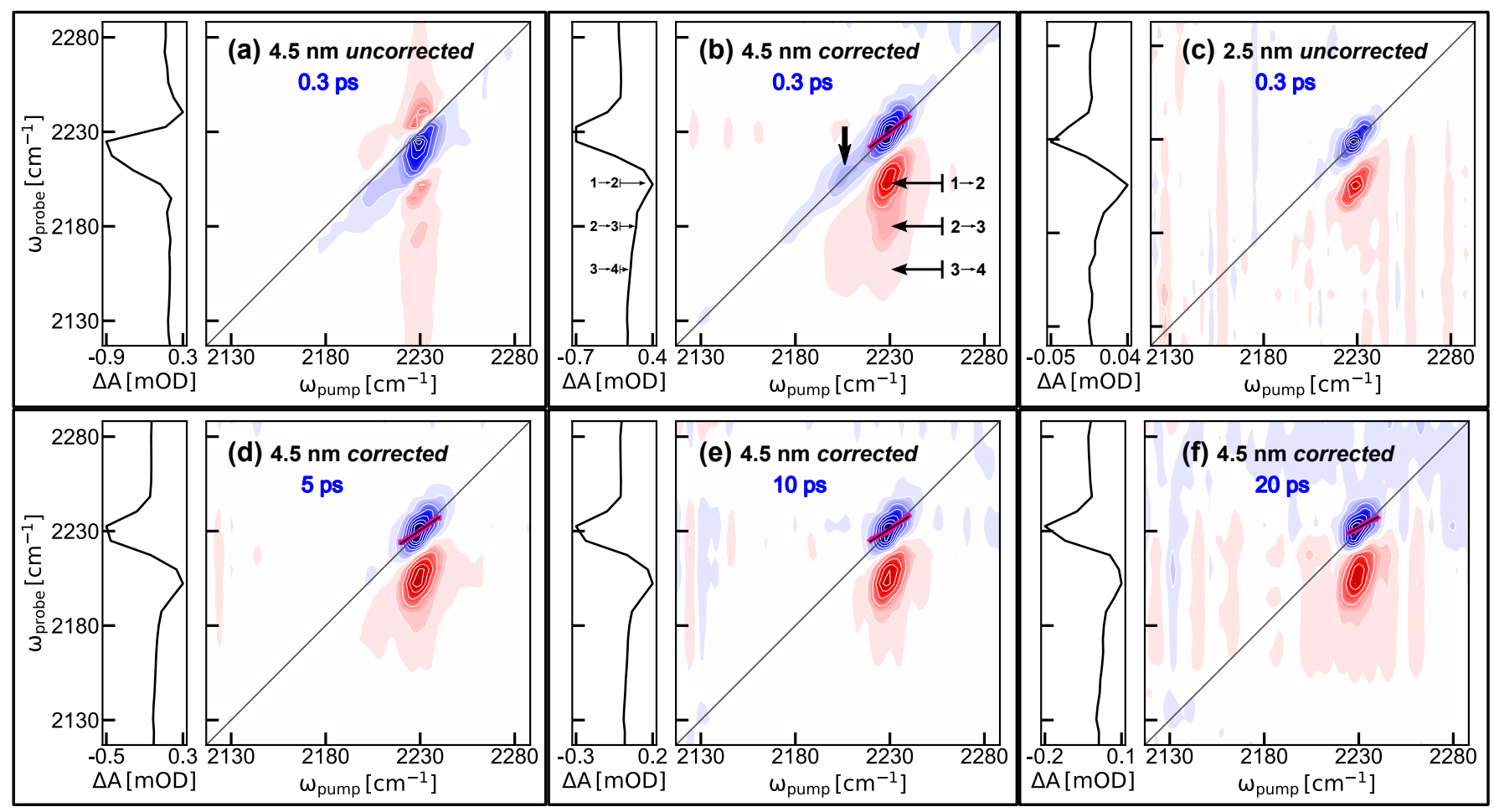

FIG. 2. 2D IR spectra at population time $0.3 \mathrm{ps}$ for (a) uncorrected $4.5 \mathrm{~nm} \mathbf{A u}-\mathbf{C N}$, (b) corrected $4.5 \mathrm{~nm} \mathbf{A u}-\mathbf{C N}$ and (c) uncorrected $2.5 \mathrm{~nm} \mathrm{Au-CN}$. Panels (d)-(f) show a time-series of corrected 2D IR spectra of $4.5 \mathrm{~nm}$ Au-CN population times $5 \mathrm{ps}, 10 \mathrm{ps}$ and $20 \mathrm{ps}$, respectively. The magenta line indicates the center of the band, and the black line a linear fit to it, from which the CLS is deduced. The labelled features are discussed in the text. Each 2D-IR spectrum is complemented with the corresponding pump-probe spectrum that has been measured independently, and phased in the same way in panels (b) and (d-f).

a positive contribution above the diagonal, while the the ESA contribution is largely missing. Applying the phasecorrection with the phase determined from the IR absorption spectrum (Fig. 1) results in the spectrum shown in Figure 2b, which closely resembles that of $2.5 \mathrm{~nm} \mathrm{Au}$ CN (Figure 2c). We observe a weak additional band at lower frequencies, labelled with an arrow, which we attribute to a site-heterogeneity on the Au surface. The elongation of the main ESA band towards lower $\omega_{\text {probe }}$ frequencies is attributed to vibrational ladder climbing due to a larger enhancement of the thicker metal layer, ${ }^{13}$ with $2 \rightarrow 3$ and $3 \rightarrow 4$ peaks showing up in addition to the normal $1 \rightarrow 2$ ESA peak. The same peaks, albeit less pronounced, are also visible in the corresponding pumpprobe spectrum (all labelled in Figure $2 \mathrm{~b}$ ).

The 2D IR spectrum in Figure 2b is elongated along the diagonal as a result of inhomogeneous broadening on the sputtered gold surface. The center line slope (CLS) of the GSB signal, shown as black line in Figure 2b, is a commonly used measure of that tilt. ${ }^{45}$ Figure 2d-f show a time-series of 2D IR spectra, evidencing that the CLS decreases as a function of time. That result is distilled out in Fig. 3, which shows the CLS as a function of population time. A single exponential fit reveals a timescale of $56 \mathrm{ps}$ for spectral diffusion, in good agreement of previous results on a thinner Au layer. ${ }^{12}$ No solvent has been

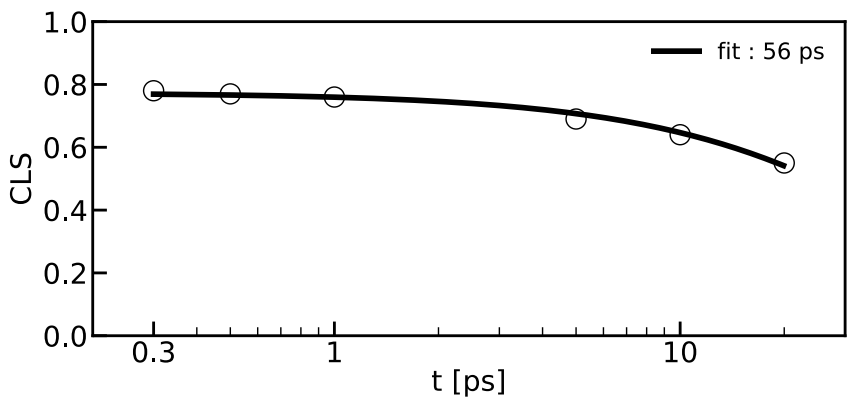

FIG. 3. Center line slope (CLS) as a function of population time of the corrected 2D IR spectra on $4.5 \mathrm{~nm}$ Au-CN. The solid line shows a single exponential fit to the data.

present in these experiments, hence spectral diffusion reflects the mobility of the molecules on the surface.

Phase corrections are not uncommon in various forms of spectroscopy. One may distinguish between situations, in which a phase correction becomes necessary due to a "lack of knowledge", and others, in which one corrects for an inherent physical effect. One example for the first case is NMR spectroscopy, where spectra are routinely "phased" to obtain symmetric lineshapes, to compensate for the unknown phase of the measured sig- 


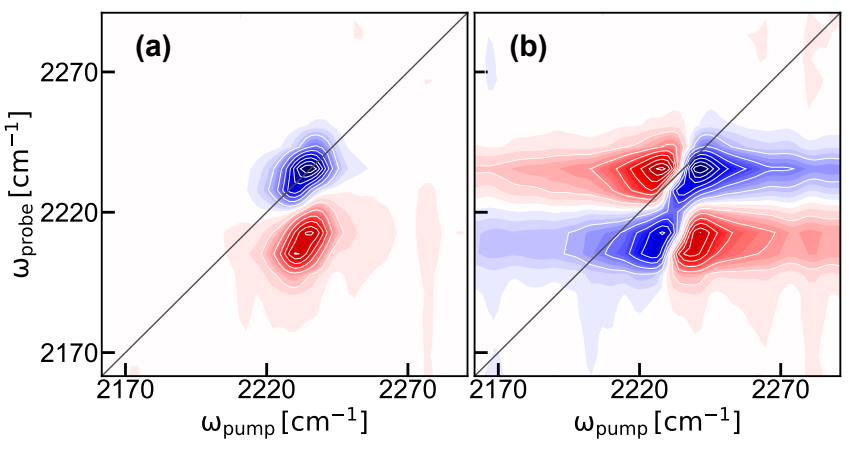

FIG. 4. (a) 2D IR spectrum $p$-PhCN in ethanol solution, measured in pump-probe geometry, and (b) the same spectrum wrongly phased with phase offset of $\pi / 2$.

nal vs. the local oscillator in the electronics of the NMR spectrometer. ${ }^{46}$ In the context of $2 \mathrm{D}$ IR spectroscopy, phasing is a major concern for instruments utilizing a box-CARS geometry, in which beam paths of typically $1 \mathrm{~m}$ length need to be known with $\sim 100 \mathrm{~nm}$ absolute precision. To phase such spectra, one typically makes use of the "projection slice theorem", ${ }^{47}$ and tries to match an independently measured pump-probe spectrum with the projection of the $2 \mathrm{D}$ IR spectrum onto the $\omega_{\text {probe }}$ axis in dependence of a phase factor that is added to the latter. This procedure does not work here, since a pump-probe spectrum are equally lineshape-distorted, see Fig. 2a. Also the pump-probe spectra consists of two contributions, GSB/SE and ESA, which typically strongly overlap spectrally, and the overlay of the two do not reveal a symmetric lineshape. Direct application of Eq. 4 would not work, which is why we had to use the absorption spectrum with a single line as a reference to determine the phase.

Note that a wrongly phased 2D spectrum measured in pump-probe geometry ${ }^{42-44}$ is distorted differently, see Fig. 4. When the pump-pulse pair is generated in an interferometer, also these spectra need to be phased with a phase factor determined from the interferogram of the two pump-pulses. ${ }^{44}$ Fig. 4 a shows the correctly phased 2D IR spectrum of $p$ - $\mathrm{PhCN}$ in ethanol solution (without any concern regarding any intrinsic lineshape distortions), and Fig. 4b when off-setting this phase by $\pi / 2$. Since the phase is added to the pump-pulse pair, the resulting 2D IR spectrum is distorted along the $\omega_{\text {pump }}$-axis, in contrast to the $\omega_{\text {probe }}$-axis in Fig. $2 \mathrm{a}$.

SEIRA spectroscopy, as well as the resonance Mie mechanism, ${ }^{30}$ are among the examples, where the phaseshift leading to distorted lineshapes is an inherent effect. For 1D spectra, correction schemes similar to the one applied here have been suggested. ${ }^{30,31}$ However, to the best of our knowledge, the same idea has not been extended to 2D spectroscopy. Here, we introduced such a phenomenological correction scheme for lineshape distorted 2D IR spectra of a metal-adsorbate system, utilizing one universal and frequency-independent phase factor that is determined by symmetrizing its IR absorption spectrum. 2D IR spectra are revealed that can be interpreted very intuitively in exactly the same way as "conventional" 2D IR spectra in the solution phase. Even though our correction scheme was demonstrated for sputtered metal layers, it should also apply to nanoantenna systems. In this regard, the phase lag function described in Refs. 14, 24, and 48 should, in principle, be equivalent to the phase correction factor used here. It has been argued that the degree to which plasmonic effects produce Fano lineshapes should correlate with a change in dephasing dynamics, ${ }^{48}$ however, we see in Fig. 1b that the bandwidth after phase correction is the same for all layer thicknesses within signal-to-noise $\left(\sim 13 \mathrm{~cm}^{-1}\right)$. Also with respect to population relaxation $\left(T_{1}\right)$, the same timescales are found for undistorted and lineshape distorted spectra, see Fig. S4, in agreement with the observation made in Ref. 49.

Here, we demonstrated the approach for a single isolated line without substructure. For more congested spectra, or, if an absorption line has substructure e.g. due to hydrogen bonding, direct application of Eq. 4 will not work. In this case, we propose to use a proper reference sample with a single line to determine the phase, and then apply that phase to the more complicated spectrum; Fig. S1d emphasizes that this will work in a pretty wide frequency range. We have verified that the phase reproducibility is $\sim 0.05 \mathrm{rad}$, small enough to have negligible effect on the corrected 2D IR spectra, provided the substrates have been sputtered in the same run.

Fano-like lineshapes are widespread, and occur under very different physical situations. The correction scheme introduced here should be applicable regardless of the underlying coupling mechanism, and thus extends the scope of 2D IR spectroscopy significantly.

\section{MATERIALS AND METHODS}

$\mathrm{CaF}_{2}$ windows were sputter-coated with ultrathin layers of $\mathrm{Au}$ (Baltic Präparation) using an $\mathrm{Ar}^{+}$-ion sputter coater (Safematic CCU-010 HV) under the following conditions: working distance $7 \mathrm{~cm}$, current $25 \mathrm{~mA}$, pressure $8 \cdot 10^{-5}$ mbar and Ar pressure of $5 \cdot 10^{-2} \mathrm{mbar}$, resulting in the sputtering rate of $0.02-0.03 \mathrm{~nm} / \mathrm{s}$. The average thickness (mass thickness) of the Au layers were monitored with a quartz microbalance. Au coated $\mathrm{CaF}_{2}$ windows were incubated with solutions of $1 \mathrm{mM} 4$ Mercaptobenzonitrile (Santa Cruz Biotechnology, Inc.) in spectroscopic grade ethanol for $12 \mathrm{~h}$, and subsequently rinsed thoroughly with double distilled water and ethanol and dried in a flow of nitrogen gas. All spectra were measured as dry sample.

IR absorption spectra were acquired with a commercial FTIR spectrometer (Bruker VERTEX 80V) with $4 \mathrm{~cm}^{-1}$ spectral resolution. Reference spectra were taken with a bare $\mathrm{CaF}_{2}$ window.

The ultrafast 2D IR setup was described in detail elsewhere. ${ }^{44}$ Briefly, 100 fs short mid-IR pulses centered at $2230 \mathrm{~cm}^{-1}$ were generated in an $\mathrm{OPA}^{50}$ pumped with a $5 \mathrm{kHz}$ amplified Ti-Sapphire laser system (Spec- 
tra Physics, Spitfire). The output from the OPA was split into pump, probe and reference beams with a $\mathrm{BaF}_{2}$ wedge. The pump beam entered a Mach-Zehnder interferometer to create collinear pump pulse pairs. All beams were then collected with an off-axis concave mirror and directed to the sample in p-polarization. To avoid sample degradation, the sample position was adjusted to be out of focus, which resulted in a fluence of $0.44 \mathrm{~J} / \mathrm{m}^{2}$ $\left(\varnothing_{\text {pump }}=925 \mu \mathrm{m}\right)$. Probe and reference beams were spectrally dispersed and imaged with a spectrograph (JobinYvon Triax, 150 lines $\mathrm{mm}^{-1}$ grating) onto a 2x32 pixel MCT array detector (Infrared Associates) for detection with a resolution of $\sim 6 \mathrm{~cm}^{-1}\left(\omega_{\text {probe }}\right)$. To suppress scattering from the metal layer, a quasi-phase cycling scheme was employed by placing a wobbling ZnSe window, introduced in the pump path at Brewster angle (after the interferometer). ${ }^{51}$ The $2 \mathrm{D}$ IR spectra were acquired by fast scanning the coherence time (delay between the two pump pulses) up to $3.5 \mathrm{ps}$ with a resolution of $\sim 1.93$ $\mathrm{cm}^{-1}\left(\omega_{\text {pump }}\right)$. The earliest 2D IR spectrum considered was measured with a population time of $300 \mathrm{fs}$, in order to avoid pump-probe overlap.

In the correction step of $2 \mathrm{D}$ IR spectra, $\tilde{A}(t)$ in Eq. 3 had to be apodized with a cosine function in order to eliminate truncation artifacts.

Supporting Information: Additional IR absorption and 2D IR spectra (Figures S1-S3). Pump-probe data (Figure S4).

Acknowledgments: The authors thank Jan Helbing for helpful discussions and the Center for Microscopy and Image Analysis (University of Zurich) for providing the sputter coating facility. The research was supported by the Swiss National Science Foundation (grant CRSII2_160801/1) and the University Research Priority Program (URPP) for solar light to chemical energy conversion (LightChEC) of the University of Zurich.

\section{References:}

(1) Andersen, P. C.; Rowlen, K. L. Appl. Spectrosc. 2002, $56,124 \mathrm{~A}-135 \mathrm{~A}$.

(2) Osawa, M.; Ataka, K. Surf. Sci. 1992, 262, L118-L122.

(3) Neubrech, F.; Huck, C.; Weber, K.; Pucci, A.; Giessen, H. Chem. Rev. 2017, 117, 5110-5145.

(4) Hartstein, A.; Kirtley, J. R.; Tsang, J. C. Phys. Rev. Lett. 1980, 45, 201-204.

(5) Yoshida, S.; Yamaguchi, T.; Kinbara, A. J. Opt. Soc. Am. 1971, 61, 62.

(6) Osawa, M.; Ikeda, M. J. Phys. Chem. 1991, 95, 99149919.

(7) Adato, R.; Aksu, S.; Altug, H. Mater. Today 2015, 18, 436-446.

(8) Selig, O.; Siffels, R.; Rezus, Y. L. A. Phys. Rev. Lett. 2015, 114, 233004.

(9) Gandman, A.; Mackin, R. T.; Cohn, B.; Rubtsov, I. V.; Chuntonov, L. ACS Nano 2018, 12, 4521-4528.

(10) Neubrech, F.; Pucci, A.; Cornelius, T. W.; Karim, S.;
García-Etxarri, A.; Aizpurua, J. Phys. Rev. Lett. 2008, 101, 157403.

(11) Kraack, J. P.; Lotti, D.; Hamm, P. J. Chem. Phys. 2015, 142, 212413.

(12) Kraack, J. P.; Lotti, D.; Hamm, P. Proc. SPIE 2015, 95490S.

(13) Kraack, J. P.; Kaech, A.; Hamm, P. J. Phys. Chem. C 2016, 120, 3350-3359.

(14) Gandman, A.; Mackin, R.; Cohn, B.; Rubtsov, I. V.; Chuntonov, L. J. Phys. Chem. Lett. 2017, 8, 3341-3346.

(15) Fano, U. Phys. Rev. 1961, 124, 1866-1878.

(16) Ishida, K. P.; Griffiths, P. R. Anal. Chem. 1994, 66, 522-530.

(17) Bjerke, A. E.; Griffiths, P. R.; Theiss, W. Anal. Chem. 1999, 71, 1967-1974.

(18) Krauth, O.; Fahsold, G.; Pucci, A. J. Chem. Phys. 1999, 110, 3113-3117.

(19) Priebe, A.; Sinther, M.; Fahsold, G.; Pucci, A. J. Chem. Phys. 2003, 119, 4887-4890.

(20) Osawa, M.; Ataka, K.-i.; Yoshii, K.; Nishikawa, Y. Appl. Spectrosc. 1993, 47, 1497-1502.

(21) Evenschor, M.; Grosse, P.; Theiss, W. Vib. Spectrosc. 1990, 1, 173-177.

(22) Theiß, W.; Detemple, R.; Ozanam, F. Am. Inst. Phys. Conf. Proc 1998, 586-589.

(23) Rezus, Y. L. A.; Selig, O. Opt. Express. 2016, 24, 1220212227.

(24) Mackin, R. T.; Cohn, B.; Chuntonov, L.; Rubtsov, I. V. In Coherent Multidimensional Spectroscopy; Cho, M., Ed.; Springer Singapore: Singapore, 2019; pp 287-310.

(25) Langreth, D. C. Phys. Rev. Lett. 1985, 54, 126-129.

(26) Chabal, Phys. Rev. Lett. 1985, 55, 845-848.

(27) Tautz, F. S. Prog. Surf. Sci. 2007, 82, 479-520.

(28) Braatz, C. R.; Öhl, G.; Jakob, P. J. Chem. Phys. 2012, 136, 134706.

(29) Koitaya, T.; Shiozawa, Y.; Mukai, K.; Yoshimoto, S.; Yoshinobu, J. J. Chem. Phys. 2016, 144, 054703.

(30) Miljković, M.; Bird, B.; Diem, M. Analyst 2012, 137, 3954-3964.

(31) Ott, C.; Kaldun, A.; Raith, P.; Meyer, K.; Laux, M.; Evers, J.; Keitel, C. H.; Greene, C. H.; Pfeifer, T. Science 2013, 340, 716-720.

(32) Hamm, P.; Zanni, M. Concepts and Methods of 2D Infrared Spectroscopy; Cambridge University Press: Cambridge, 2011.

(33) Kraack, J. P.; Hamm, P. PCCP 2016, 18, 16088-16093.

(34) Paleček, D.; Tek, G.; Lan, J.; Iannuzzi, M.; Hamm, P. J. Phys. Chem. Lett. 2018, 9, 1254-1259.

(35) Finkelstein-Shapiro, D.; Pullerits, T.; Hansen, T. J. Chem. Phys. 2018, 148, 184201.

(36) Hisatomi, T.; Kubota, J.; Domen, K. Chem. Soc. Rev. 2014, 43, 7520-7535.

(37) Kraack, J. P.; Hamm, P. Chem. Rev. 2017, 117, 1062310664.

(38) Laor, U.; Schatz, G. C. J. Chem. Phys. 1981, 82, 566570.

(39) Bertie, J. E.; Zhang, S. L. Can. J. Chem. 1992, 70, 520531.

(40) Gersten, J. I.; Nitzan, A. Surf. Sci. 1985, 158, 165-189.

(41) Osawa, M. Bull. Chem. Soc. Jpn. 1997, 70, 2861-2880.

(42) DeFlores, L. P.; Nicodemus, R. A.; Tokmakoff, A. Opt. Lett. 2007, 32, 2966-2968.

(43) Shim, S. H.; Zanni, M. T. Phys. Chem. Chem. Phys. 
2009, 11, 748-761.

(44) Helbing, J.; Hamm, P. J. Opt. Soc. Am. B 2011, 28, 171.

(45) Kwak, K.; Park, S.; Finkelstein, I. J.; Fayer, M. D. J. Chem. Phys. 2007, 127, 124503.

(46) Keeler, J. Understanding NMR Spectroscopy (2004); Department of Chemistry, Univerrsity of Cambridge, 2016.

(47) Gallagher Faeder, S. M.; Jonas, D. M. J. Phys. Chem. A 1999, 103, 10489-10505.

(48) Metzger, B.; Muller, E.; Nishida, J.; Pollard, B.; Hentschel, M.; Raschke, M. B. Phys. Rev. Lett. 2019, $123,153001$.

(49) Cohn, B.; Prasad, A. K.; Chuntonov, L. J. Chem. Phys. 2018, 148, 1-5.

(50) Hamm, P.; Kaindl, R. A.; Stenger, J. Opt. Lett. 2000, 25, 1798-1800.

(51) Bloem, R.; Garrett-Roe, S.; Strzalka, H.; Hamm, P.; Donaldson, P. Opt. Express. 2010, 18, 27067-27078. 